\title{
High thermoelectric and flexible PEDOT/SWCNT/BC nanoporous film derived from aerogels
}

\author{
Fang Jia, ${ }^{\dagger}$ Ruili Wu, ${ }^{\dagger}$ Chan Liu, ${ }^{+}$Jin-le Lan, ${ }^{* \dagger}$ Yuan-hua Lin $*,+$ \\ Xiaoping Yang ${ }^{\dagger}$
}

$+\quad$ State Key Laboratory of Organic-Inorganic Composites, Beijing University of Chemical Technology, North Third Ring Road 15, Chaoyang District, Beijing 100029, P. R. China

$\$$ State Key Laboratory of New Ceramics and Fine Processing, School of Materials Science and Engineering, Tsinghua University, Shuangqing Poad 30, Haidian District, Beijing 100084, P. R. China

*Correspondingauthor:lanjl@mail.buct.edu.cn,linyh@mail.tsinghua.edu.cn

Total number of pages: 13

Total number if figures: 5

Total number of tables: 2 


\section{Table of Content}

Figure S1. SEM images of pristine BC nanofibers and PEDOT/BC composite at different reaction time (24h, 39h, 42h, 45h, and 48h).

Figure S2. SEM images of PEDOT/BC composite prepared at different $\mathrm{Fe}^{3+} / \mathrm{EDOT}$ molar ratio(1, $1.2,1.6,1.8,2.2,2.6)$. S4

Figure S3. Raman spectra of PEDOT/BC films with different reaction time. S5

Figure S4. FTIR spectra of BC, PEDOT/BC, PEDOT/SWCNT/BC films. S7 Figure S5. Thermoelectric properties of PEDOT/BC films with different reaction time and different $\mathrm{Fe}^{3+} / \mathrm{EDOT}$ molar ratio S8

Eq S1and S2. The equation of parallel-connected models $\mathrm{S} 10$

Table S1. Density data of PEDOT/BC and PEDOT/SWCNT/BC films S11

Table S2. The thermal conductivity parameters of the PEDOT/BC and PEDOT/SWCNT/BC films 


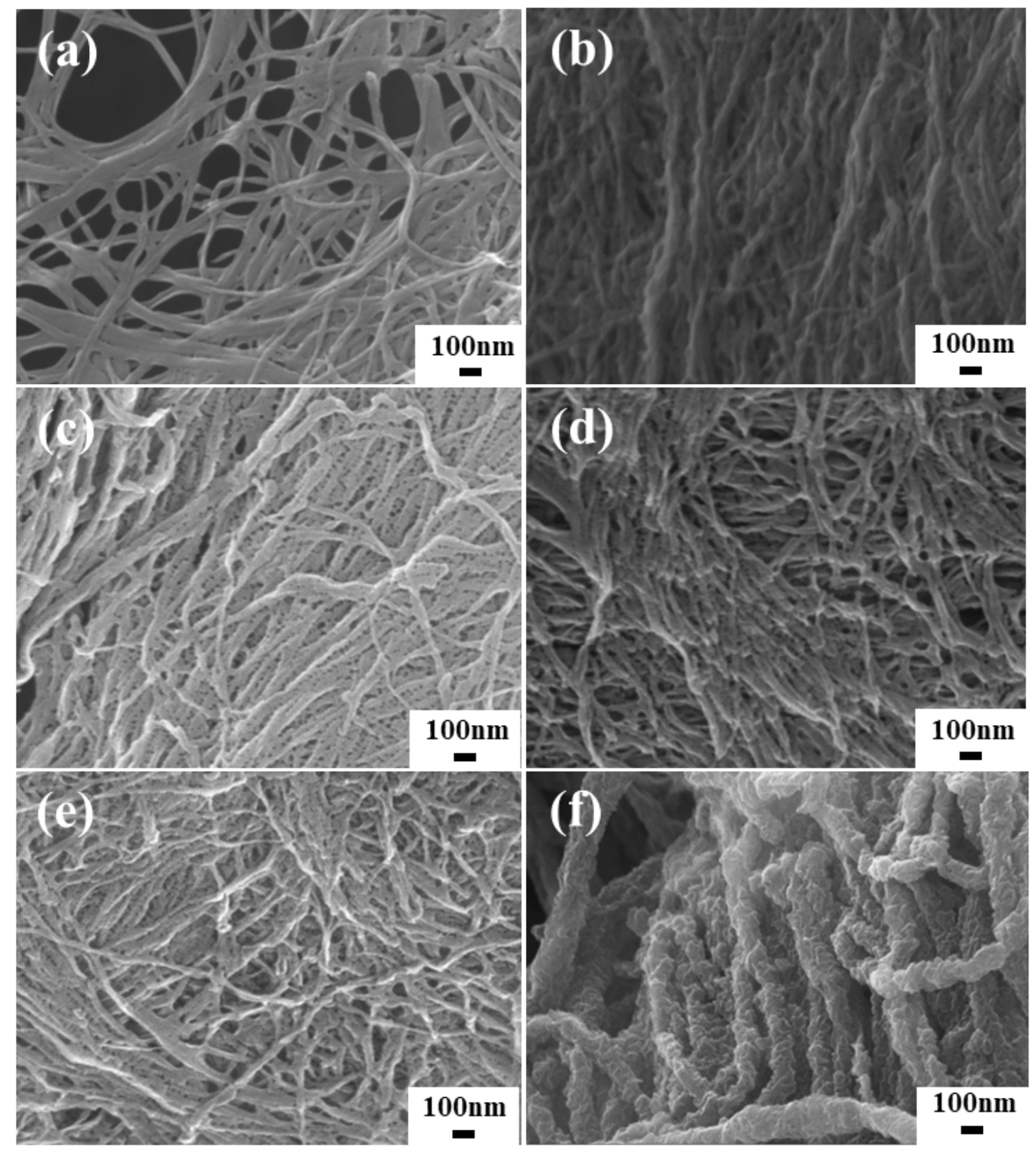

Figure S1. SEM images of pristine BC nanofibers (a). PEDOT/BC composite prepared with different reaction time: $24 \mathrm{~h}(\mathrm{~b}), 39 \mathrm{~h}(\mathrm{c}), 42 \mathrm{~h}(\mathrm{~d}), 45 \mathrm{~h}(\mathrm{e})$, and $48 \mathrm{~h}(\mathrm{f})$. 


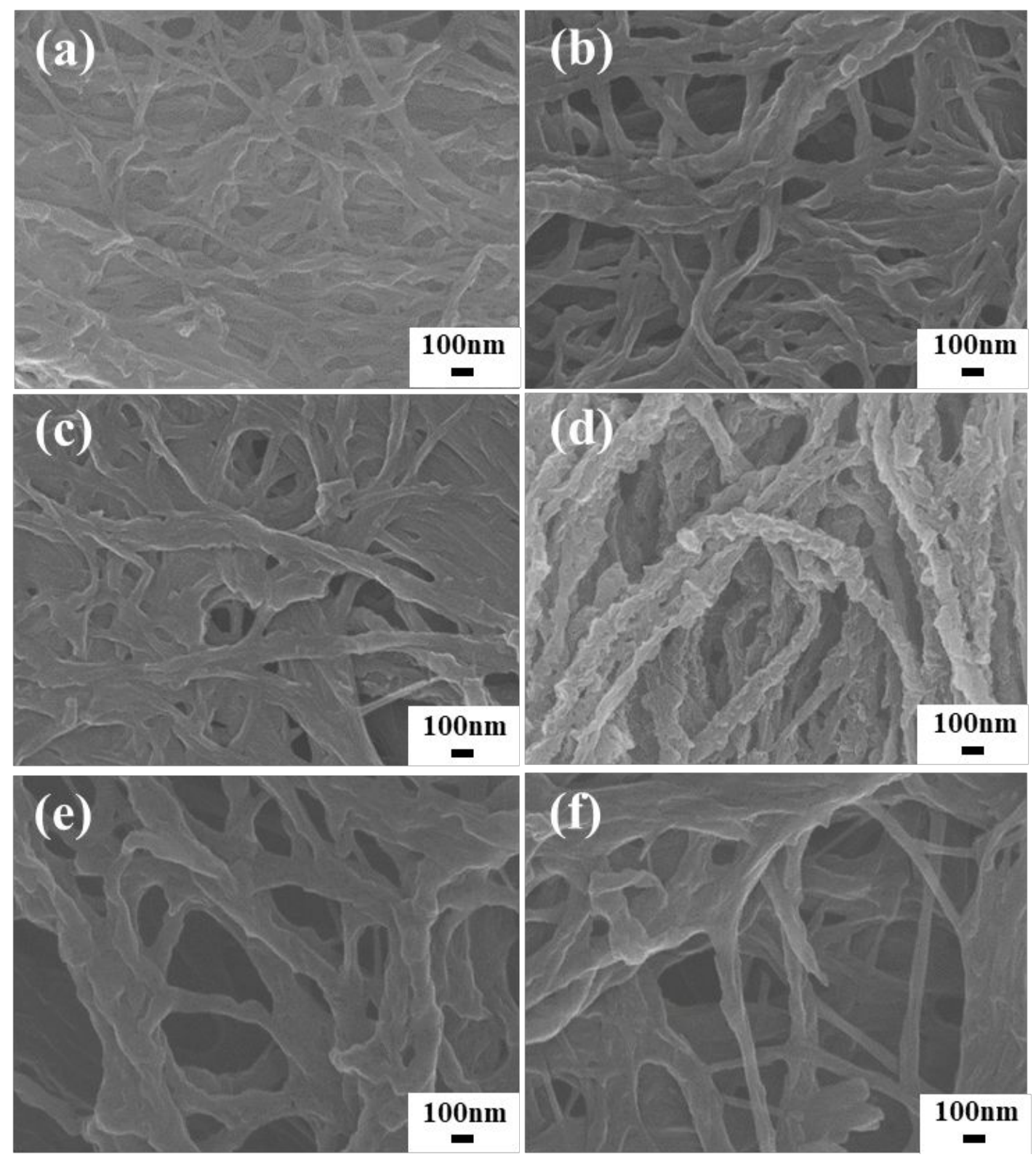

Figure S2. SEM images of PEDOT/BC composite prepared at different $\mathrm{Fe}^{3+} / \mathrm{EDOT}$ molar ratio: $\quad \mathrm{Fe}^{3+} / \mathrm{EDOT}=1(\mathrm{a}), \quad \mathrm{Fe}^{3+} / \mathrm{EDOT}=1.2(\mathrm{~b}), \quad \mathrm{Fe}^{3+} / \mathrm{EDOT}=1.6(\mathrm{c})$, $\mathrm{Fe}^{3+} / \mathrm{EDOT}=1.8(\mathrm{~d}), \mathrm{Fe}^{3+} / \mathrm{EDOT}=2.2(\mathrm{e}), \mathrm{Fe}^{3+} / \mathrm{EDOT}=2.6(\mathrm{f})$. 


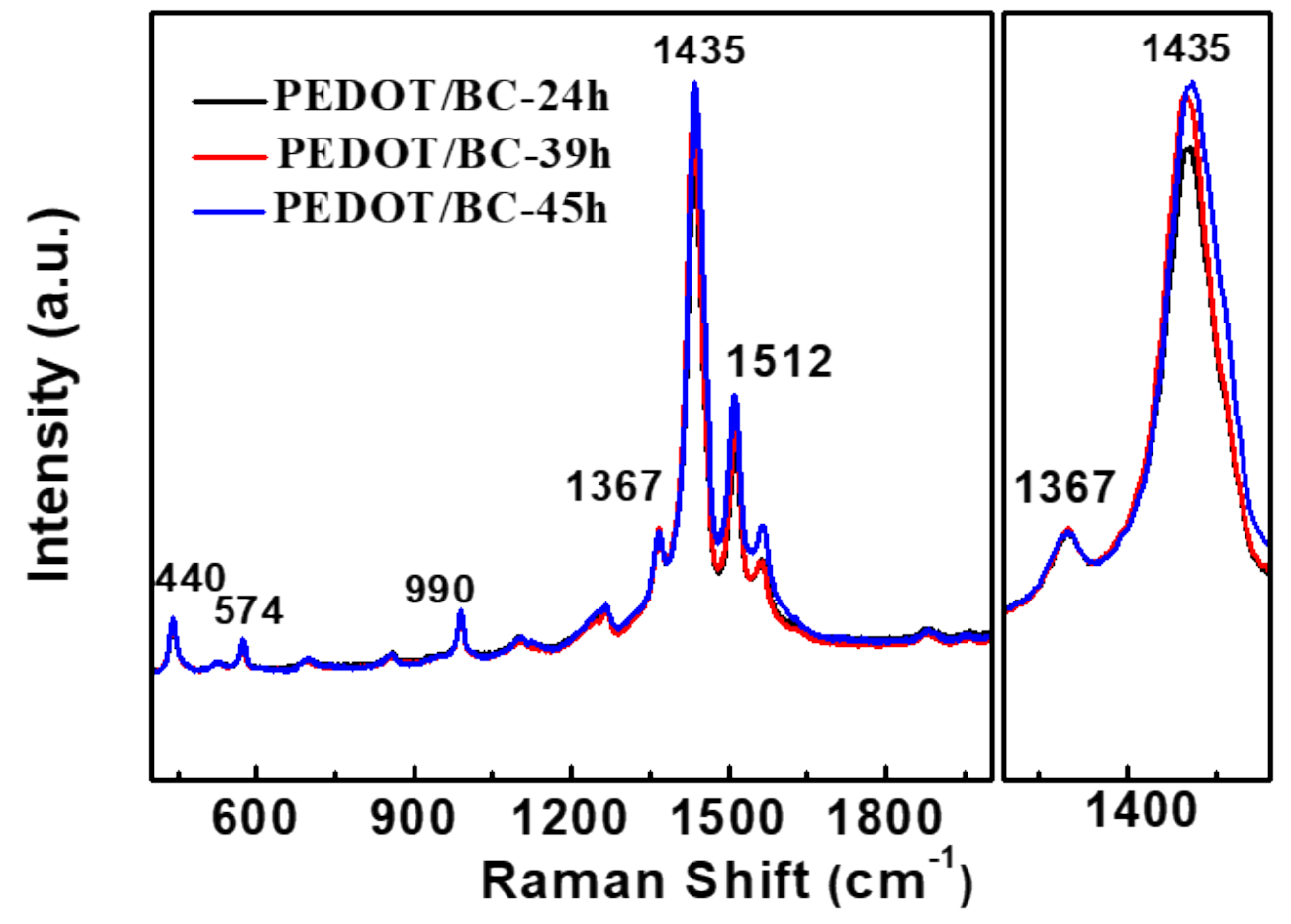

Figure S3. Raman spectra of PEDOT/BC films with different reaction time.

Figure. S3 presented the Raman spectra of PEDOT/BC films with the different polymerization time. Three peaks at 440,574 and $990 \mathrm{~cm}^{-1}$ represent the oxyethylene ring deformation, the $\mathrm{C}_{\beta}-\mathrm{C}_{\beta}$ stretching $\left(1367 \mathrm{~cm}^{-1}\right)$, symmetrical $\mathrm{C}_{\alpha}=\mathrm{C}_{\beta}(-\mathrm{O})$ stretching $\left(1435 \mathrm{~cm}^{-1}\right)$, asymmetrical $\mathrm{C}=\mathrm{C}$ stretching $\left(1512 \mathrm{~cm}^{-1}\right)$ are observed in all the sample. The peaks at 1367 and $1435 \mathrm{~cm}^{-1}$ reflect the benzenoid structure of the PEDOT chain. The change of doping level of the PEDOT chain can change the quinoid/benzenoid ratio. ${ }^{1}$ Meanwhile, the structure of oxyethylene rings of PEDOT does not change with the doping states, so that we are able to normalize the peak with a reference peak of $990 \mathrm{~cm}^{-1}$, which is used to represent the same amount of thiophene units. After normalization, the peak intensities at 1367 and $1435 \mathrm{~cm}^{-1}$ reflect percentage of the benzenoid-type 
structure. ${ }^{2}$ The peak intensities at 1367 and $1435 \mathrm{~cm}^{-1}$ are greater at $45 \mathrm{~h}$ reaction time than $29 \mathrm{~h}$ and $45 \mathrm{~h}$, which indicated the lower doping level. 


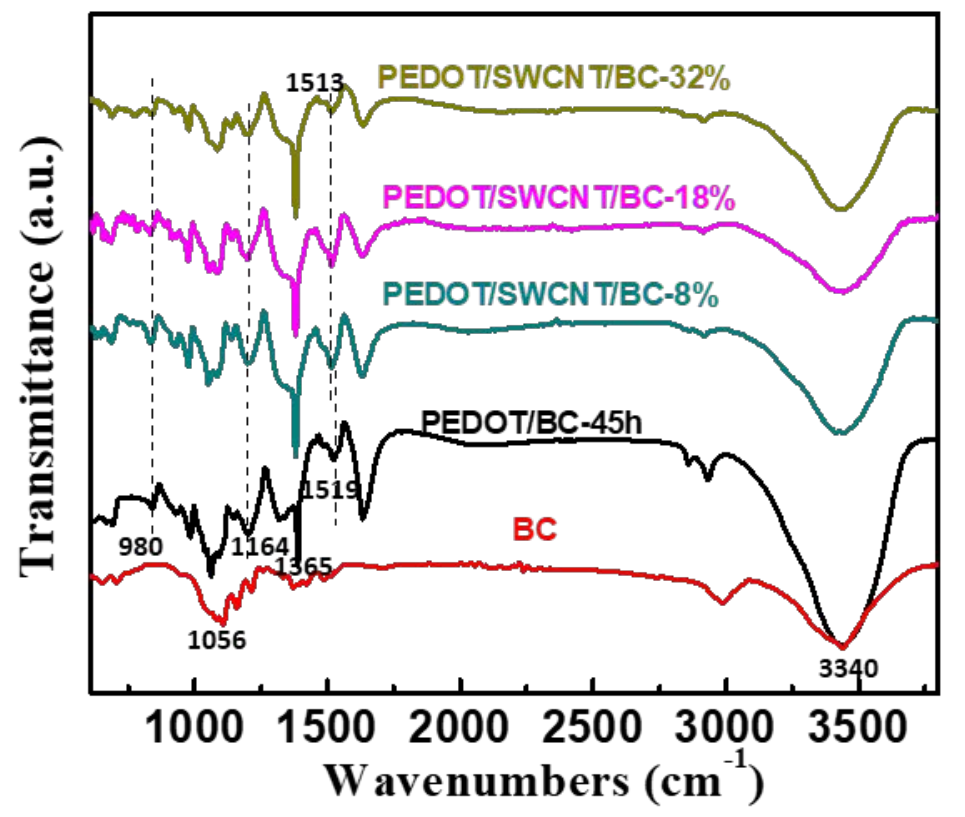

Figure S4. FTIR spectra of BC, PEDOT/BC, PEDOT/SWCNT/BC films.

The FTIR reflectance spectra of BC, PEDOT/BC and PEDOT/SWCNT/BC films are compared in Fig. S4. The obvious bands at $1056 \mathrm{~cm}^{-1}$ originate from the vibrations of $\mathrm{C}-\mathrm{O}-\mathrm{H}$ is the characteristic bands of BC. Besides, the strong bands at $3340 \mathrm{~cm}^{-1}$ derive from the stretching of $\mathrm{H}-\mathrm{O}-\mathrm{H}$ bending of the absorbed water. Compared to $\mathrm{BC}$, the FTIR reflectance spectra of PEDOT/BC film contains a group of characteristic bands at 980 $\mathrm{cm}^{-1}, 1164 \mathrm{~cm}^{-1}, 1365 \mathrm{~cm}^{-1}$ and $1519 \mathrm{~cm}^{-1}$ corresponding to C-S, C-O-C, C-C and C=C in PEDOT. Comparing the curves of PEDOT/BC and PEDOT/SWCNT/BC films, the $\mathrm{C}=\mathrm{C}$ peak of PEDOT at $1519 \mathrm{~cm}^{-1}$ shift to $1513 \mathrm{~cm}^{-1}$, which is due to the $\pi$ electron cloud move in a larger area when $\mathrm{C}=\mathrm{C}$ of PEDOT and SWCNT are close, and lower the bond energy, improve the overall stability, the absorption frequency to decrease. The FTIR reflectance spectra indicating $\pi-\pi$ conjugated interaction between SWCNT and PEDOT and the charge transfer take place in the interface. 
(a)

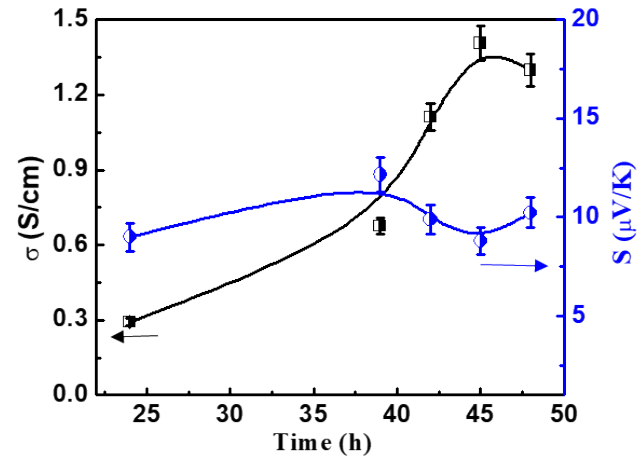

(c)

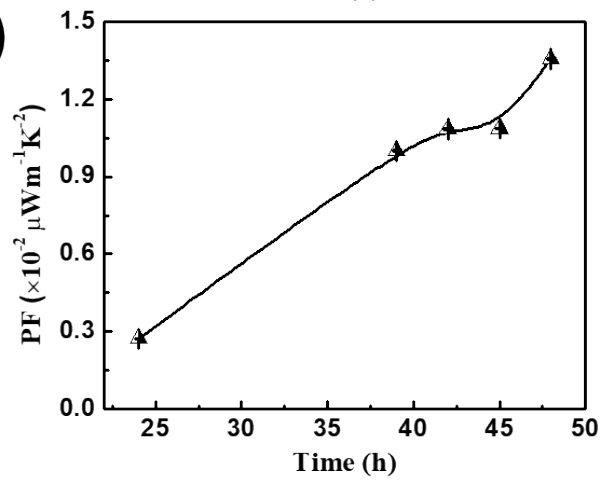

(b)

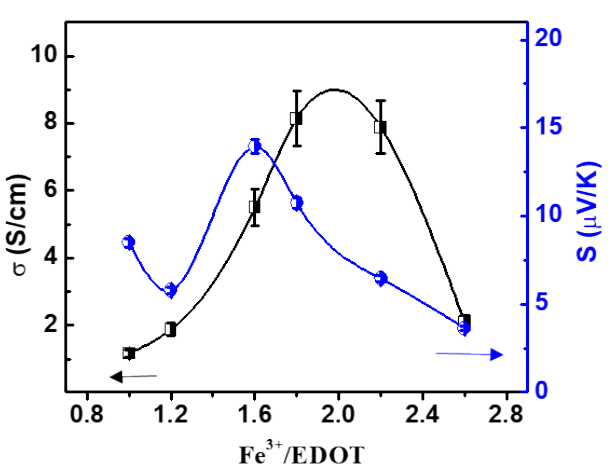

(d)

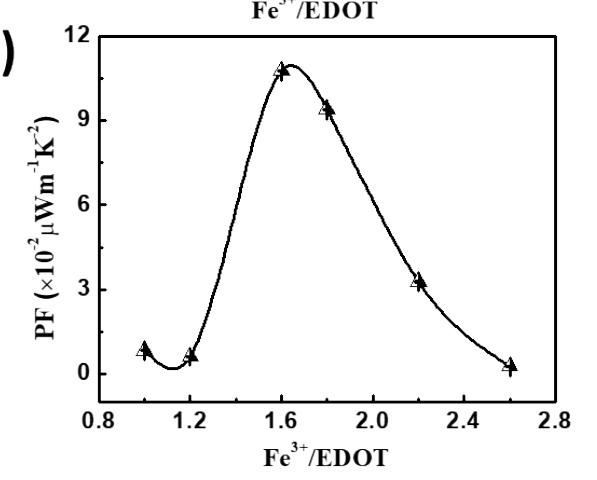

Figure S5. (a,c)Thermoelectric properties of PEDOT/BC films with different reaction time; $(b, d)$ Thermoelectric properties of PEDOT/BC films prepared at different $\mathrm{Fe}^{3+} /$ EDOT molar ratio.

The thermoelectric properties of PEDOT/BC films with different reaction time and prepared at different $\mathrm{Fe}^{3+} /$ EDOT molar ratio at room temperature as shown in Figure. S4. The conductivity increased with the reaction time before $45 \mathrm{~h}$ and then decreased (Figure. S4(a)). This could be contributed to an increase in the thickness of PEDOT layers and a decrease in the doping level as the reaction time increase (Figure.S1 and Figure. S3). During all reaction time, the Seebeck coefficient showed little change and was kept at about $10 \mu \mathrm{V} / \mathrm{K}$. Figure. S4(b) shown the thermoelectric properties of PEDOT/BC films with different molar ratio of $\mathrm{Fe}^{3+} / \mathrm{EDOT}$ at $45 \mathrm{~h}$. The largest electrical conductivity of PEDOT/BC film was $8.5 \mathrm{~S} / \mathrm{cm}$ at $\mathrm{Fe}^{3+} / \mathrm{EDOT}=1.8$. The maximum Seebeck coefficient of 
13.9 $\mu \mathrm{V} / \mathrm{K}$ and the maximum power factor of $0.11 \mu \mathrm{W} \mathrm{m} \mathrm{m}^{-1} \mathrm{~K}^{-2}$ were obtained at the $\mathrm{Fe}^{3+} / \mathrm{EDOT}=1.6($ Figure. S4(b,d)). 
Eq S1and S2: The equation of parallel-connected models

The parallel-connected models of PEDOT/SWCNT/BC composites can be written as:

$$
\begin{gathered}
\sigma_{\mathrm{p}}=\left(v_{B C}+v_{P E D O T}\right) \sigma_{P E D O T / B C}+v_{S W C N T} \sigma_{S W C N T} \\
S_{P}=\frac{\left(v_{B C}+v_{P E D O T}\right) \sigma_{P E O D T / B C} S_{P E D O T / B C}+v_{S W C N T} \sigma_{S W C N T} S_{S W C N T}}{\left(v_{B C}+v_{P E D O T}\right) \sigma_{P E O D T / B C}+v_{S W C N T} \sigma_{S W C N T}}
\end{gathered}
$$

Where $\sigma_{\mathrm{P}}, v$ and $\mathrm{S}_{\mathrm{p}}$ are the electrical conductivity of parallel-connected models, volume fraction, and the Seebeck coefficient of the parallel-connected composite of parallelconnected models. 
Table S1. Density data of PEDOT/BC and PEDOT/SWCNT/BC films: Theoretical density were calculated based on the densities of the components; Bulk densities determined by calculated from geometry and weight; porosity ratio.

Sample

Bulk density $\rho$

Theoretical density $\rho_{\mathrm{T}}$

Porosity ratio $\mathrm{P}$

$\left(\mathrm{g} / \mathrm{cm}^{3}\right) \quad\left(\mathrm{g} / \mathrm{cm}^{3}\right)$

$(\%)$

PEDOT/BC-24h

0.36

1.35

73.08

PEDOT/BC-39h

0.35

1.34

73.54

PEDOT/BC-42h

0.30

1.32

77.10

PEDOT/BC-45h

0.42

1.30

67.69

PEDOT/BC-48h

0.39

1.29

69.70

PEDOT/SWCNT/BC-6\%

0.42

1.33

68.69

PEDOT/SWCNT/BC-12\%

0.39

1.39

71.80

PEDOT/SWCNT/BC-18\%

0.53

1.41

62.17

PEDOT/SWCNT/BC-28\%

0.57

1.52

62.68

PEDOT/SWCNT/BC-32\%

0.35

1.51

76.82 
Table S2. The thermal conductivity parameters of the PEDOT/BC and PEDOT/SWCNT/BC films.

\begin{tabular}{ccccc}
\hline Sample & $\begin{array}{c}\text { Density } \\
\left(\mathbf{g ~ c m}^{-\mathbf{3}}\right)\end{array}$ & $\begin{array}{c}\text { Specific } \\
\text { heat } \\
\left(\mathbf{J ~ g}^{-1} \mathbf{K}^{-\mathbf{1}}\right)\end{array}$ & $\begin{array}{c}\text { Thermal } \\
\text { diffusivity } \\
\left(\mathbf{m m}^{\mathbf{2}} \mathbf{~ s}^{-\mathbf{1}}\right)\end{array}$ & $\begin{array}{c}\text { Thermal } \\
\text { conductivity } \\
\left(\mathbf{W ~ m}^{-1} \mathbf{K}^{-1}\right)\end{array}$ \\
\hline PEDOT/BC-1.6 & 0.42 & 1.10 & 0.127 & 0.06 \\
PEDOT/SWCNT/BC- & 0.35 & 0.93 & 0.401 & 0.13 \\
32\% & & & & \\
\hline
\end{tabular}




\section{REFERENCE}

[1] Bubnova, O.; Khan, Z. U.; Malti, A.; Braun, S.; Fahlman, M.; Berggren, M.; Crispin, $X$. Optimization of the thermoelectric figure of merit in the conducting polymer poly $(3,4-$ ethylenedioxythiophene). Nature. Mat. 2011, 10 (6), 429-433, DOI 10.1038/nmat3012.

[2] Shi, W.; Qu, S.; Chen, H.; Chen, Y.; Yao, Q.; Chen, L. One-step Synthesis and Enhanced Thermoelectric Properties of Polymer-Quantum Dot Composite Films. Angew.

Chem. Int. Ed. 2018, 57 (27), 8037-8042, DOI 10.1002/ange.201802681. 